\title{
Photonic integrated WDM cross- connects for optical metro and data center networks
}

N. Calabretta, K. Prifti, N. Tessema, X. Xue, B. Pan, et al.

N. Calabretta, K. Prifti, N. Tessema, X. Xue, B. Pan, R. Stabile, "Photonic integrated WDM cross-connects for optical metro and data center networks," Proc. SPIE 10946, Metro and Data Center Optical Networks and Short-Reach Links II, 1094603 (1 February 2019); doi: 10.1117/12.2508897 


\title{
Photonic Integrated WDM Cross-Connects for Metro and Data Center Networks
}

\author{
N. Calabretta, K. Prifti, N. Tessema, X. Xue, B. Pan, R. Stabile \\ Institute of Photonic Integration, Eindhoven University of Technology, Eindhoven, The Netherlands \\ n.calabretta@tue.nl
}

\begin{abstract}
We present a scalable and novel modular optical metro core node architecture and low cost metro access node architectures with edge computing functionalities employing photonic WDM integrated switches. Photonic integrated switches has been designed as the building blocks to realize the modular metro node architectures, namely photonic WDM switches with express and add/drop ports, photonic integrated WSS aggregation/disaggregation functions for merging/dropping the network traffic, and photonic integrated multi-cast switch (MCS), as well as bandwidth variable transceivers aggregators to achieve multi-Terabits/second operation. Moreover, a dynamic re-configurable metro-access nodes based on low-cost 2-degree photonic integrated mini-ROADMs will be discussed. The lossless photonic WDM switches are based on InP technology and employ semiconductor optical amplifiers as on -chip gain element and fast switch. The photonic WDM circuits allows to switch multiple format data signals in wavelength, space, and time for full flexibility, scalability of the interconnected network elements as well as capacity. Applications to data center interconnects and $5 \mathrm{G}$ will be discussed and experimental results reported.
\end{abstract}

Keywords: Metro networks, photonic integrated devices, semiconductor optical amplifiers, photonic switching circuits.

\section{INTRODUCTION}

Boosted by cloud, IoT, and 5G applications next generation metro networks should be able to dynamically and efficiently handle large heterogeneous data traffics [1]. Photonic integrated WDM cross-connect switch architectures with wavelength, space, and time switching operation are key building blocks to realize high performance and flexible optical networks. Moreover, the fast switching operation is beneficial for statistical multiplexing and low latency operation. Their applications range from telecom metro-access and metro core networks, in which dynamic traffic, flexibility, cost, and power consumption are key parameters, to intra data center networks, in which low latency, high capacity and high connectivity are the key parameters. The advantages of deploying optical switching technologies are multi-fold: transparency to data-rate and data-format allows for extremely high I/O bandwidth without implementing signal-dependent interfaces. Moreover, switching the data signals in the optical domain has the potential to overcome the scaling issues of electronic switches. The high capacity helps to flatten the network topology avoiding bandwidth bottleneck and large latency caused by hierarchical structures. Massive O/E/O conversions can be eliminated improving the energy-efficiency and cost-efficiency.

At the metro core network level, ultra-high capacity space division multiplexing (SDM) systems have demonstrated multi $\mathrm{Pb} / \mathrm{s}$ transmission exploiting multi-core fibers. Therefore, it is envisioned that metro network nodes should be able to do reconfigurable switching for amount of traffics carried by SDM multi-core fibers in an efficient and scalable way. Modular reconfigurable optical metro node based on add/drop mu ltiplexers (ROADM) with color-less, direction-less and contention-less (CDC) are the main candidate to transparently switch SDM systems. The main challenges in CDCROADM is the cost and complexity of the switching sub-systems as the wavelength channels count and thus the traffic scales. Therefore, novel efficient and scalable ROADM for SDM system exploiting modular architectures and low cost photonic integrated switching are currently investigated.

If the metro core network is mostly characterized by the need of ultra-high capacity based on optical circuit switches, the metro access network segment will operate in a highly heterogeneous environment characterized by the existence of multiple types of access technologies, devices, and user interactions. In the next generation 5G systems, the co-existence of applications spanning from data intensive to latency sensitive $(<5 \mathrm{~ms})$ applications will demand for more flexible and dynamic optical metro networks capable to quickly and efficiently be rearranged to adapt to the different applications 
requirements [1]. Therefore, high dynamic packet-based metro access nodes employing low cost 2-degree fast add drop multiplexers for time and wavelength statistical multiplexing have been investigated [2-4].

In this work, we present a novel modular optical metro core node architecture for SDM employing a photonic WDM cross-connect switches with express and add/drop ports, photonic integrated WSS aggregation/disaggregation functions for merging/dropping the network traffic, and photonic integrated multi-cast switch (MCS). The modular approach enables to scale the architecture in a pay-as-you-grow approach and employs photonic integrated devices for wavelength and space switching to implement the modules of the ROADM. Moreover, the novel ROADM exploits the MCF for bundled traffic with common destination, so that express ports can transparently direct the traffic without any process, saving resources and power consumption. Moreover, a low-cost 2-degree photonic integrated mini-ROADMs for dynamic re-configurable metro-access nodes in a ring topology will be presented. The photonic WDM switches are based on InP technology and employ semiconductor optical amplifiers (SOA) as on-chip gain element for lossless operation. Moreover, the fast switching time of the SOA and the high contrast ratio make the SOA a good candidate for fast packet-based switching operation. The capability of the photonic WDM switches to transparently process data signals in wavelength, space, and time allows for high dynamic, flexible, and scalable interconnected networks.

\section{METRO CORE AND ACCESS NETWORK ARCHITECTURE}

A typical example of a deployed metro-core and access network scenario is shown in Fig. 1. The metro-core network is constituted by high capacity switching nodes referred in this paper as hierarchical level 3 (HL3) nodes. The HL3 switching nodes are connected to access network mainly constituted by a network of Hierarchical level-4 (HL4) nodes. The HL4 nodes are the closest to the end users and may be connected in a different topologies, a ring topology can be an example as presented in Fig. 1. The HL4 nodes can for instance support traffic for business VPN, residential network connectivity and cellular networks. The photonic WDM switches embedded in HL3 and HL4 nodes enable leveraging wavelengths to traffic conditions where they are most needed temporarily, for instance a cellular network in a stadium during sporting events. Given the amount of nodes and the effective traffic, low-cost and low power consumption is important metric especially for the metro-access segment. Several recent works in this topic are presented in [5-8]. Moreover, given the 5G requirements in terms of latency, the metro access node will be equipped with computing functionalities (edge computing nodes) as well. We will discuss more in a details the metro core node and metro access node architectures and building blocks in the next sections.

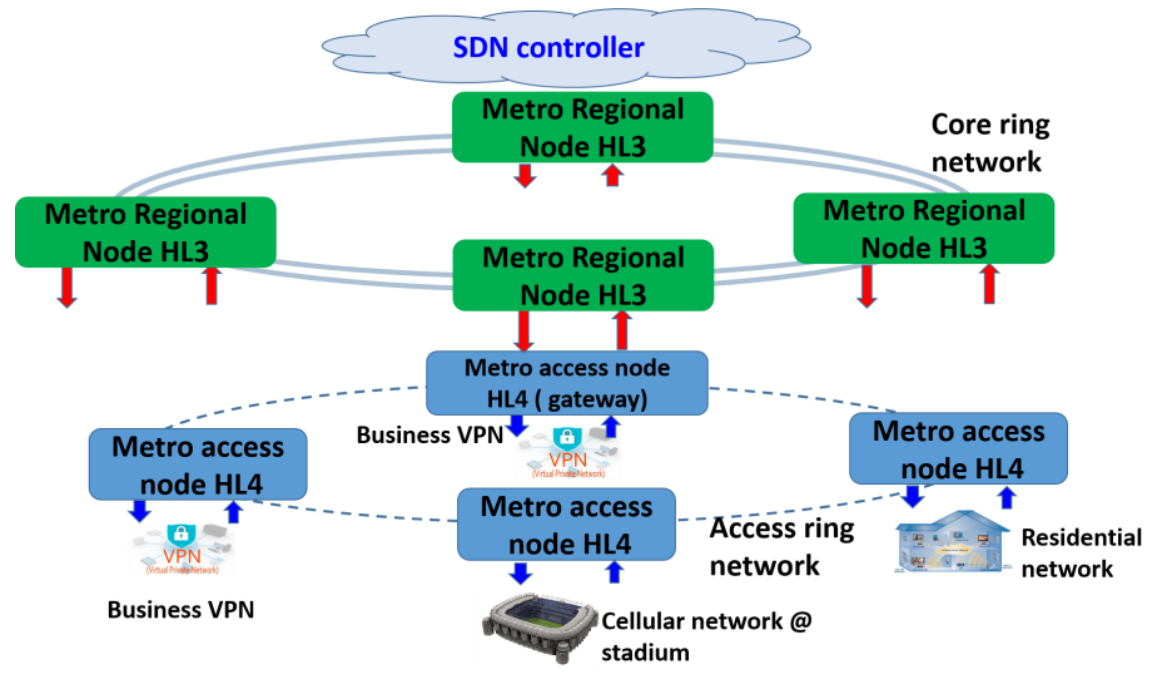

Figure 1. SDN enabled Metro core and metro access network architecture.

\subsection{Metro Core Node architecture and operation}

In this work, we demonstrate a novel modular ROADM architecture for SDM employing a photonic space switch with express (bundled traffic) and add/drop ports, photonic integrated wavelength selective switches (WSS) 
aggregation/disaggregation functions for merging/dropping the network traffic, and photonic integrated multi-cast switch (MCS). The modular approach enables to scale the architecture in a pay-as-you-grow approach and employs photonic integrated devices for wavelength and space switching to implement the modules of the ROADM. Moreover, the novel ROADM exploits the MCF for bundled traffic with common destination by disaggregating first specific traffic and then aggregates the channels for bundling traffic to same destination, so that at the next node, express ports can transparently direct the traffic without any process, saving resources and power consumption.

The proposed modular ROADM for SDM system is shown in Fig. 2. The ROADM node consists of a photonic space switch matrix (PSM) providing connectivity between the input and output multicore fibers (MCFs). Some of the ports of the PSM directly connect the input/output MCFs (express ports), while other ports are used to drop and to add traffics. The drop ports are connected to the aggregation/ disaggregation switch which separates the wavelength sets to be dropped to the local end users and those which are aggregated and added via the Add switch to the express traffic. In other words, the Agg/disaggregate switch bundles traffic that goes to the Add switch and to the MCS based on their wavelengths. Therefore, this block is implemented using simple $1 \times 2$ WSS. By bundling traffic to the same destination, efficient utilization of resources (ports of PSM, and MCF) is realized. The traffic that has to be dropped to local endusers is directed to the MCS which enables contention-less operation (via its broadcast and select feature) as shown in Fig. 2. In this way, any of the available receivers in the coherent receiver modules (CRM) can be used to receive any wavelength leading to efficient utlization of the receivers. For adding the traffic, bandwidth variable transmitter (BVT) provides the capacity to variably allocate the bandwidth of locally generated traffic at the particular node. The locally generated traffic and aggregated traffic are merged as added traffic by the Add switch. The Add switch is based on Madd $\times 1$ WSS, where Madd is its number of input ports. In the event of contention, the Add switch sends multiple traffic flows in the same wavelength channel to separate output ports. Then, the traffic of the Add switch is inserted into the network via the PSM add ports. Due to bundle at next node the aggregated traffic goes transparently through the express port, saving resources since no processing of this traffic is needed. The scalablity of the SDM ROADM node is ensured by a modular approach in a "pay as you grow" principle, where new more modules are added to accomodate the increase in traffic. The use of software defined networking (SDN) control plane provides programmability of components of the node to achieve the level of flexiblity, channel bandwidth, path, and energy requirement of the metro network.

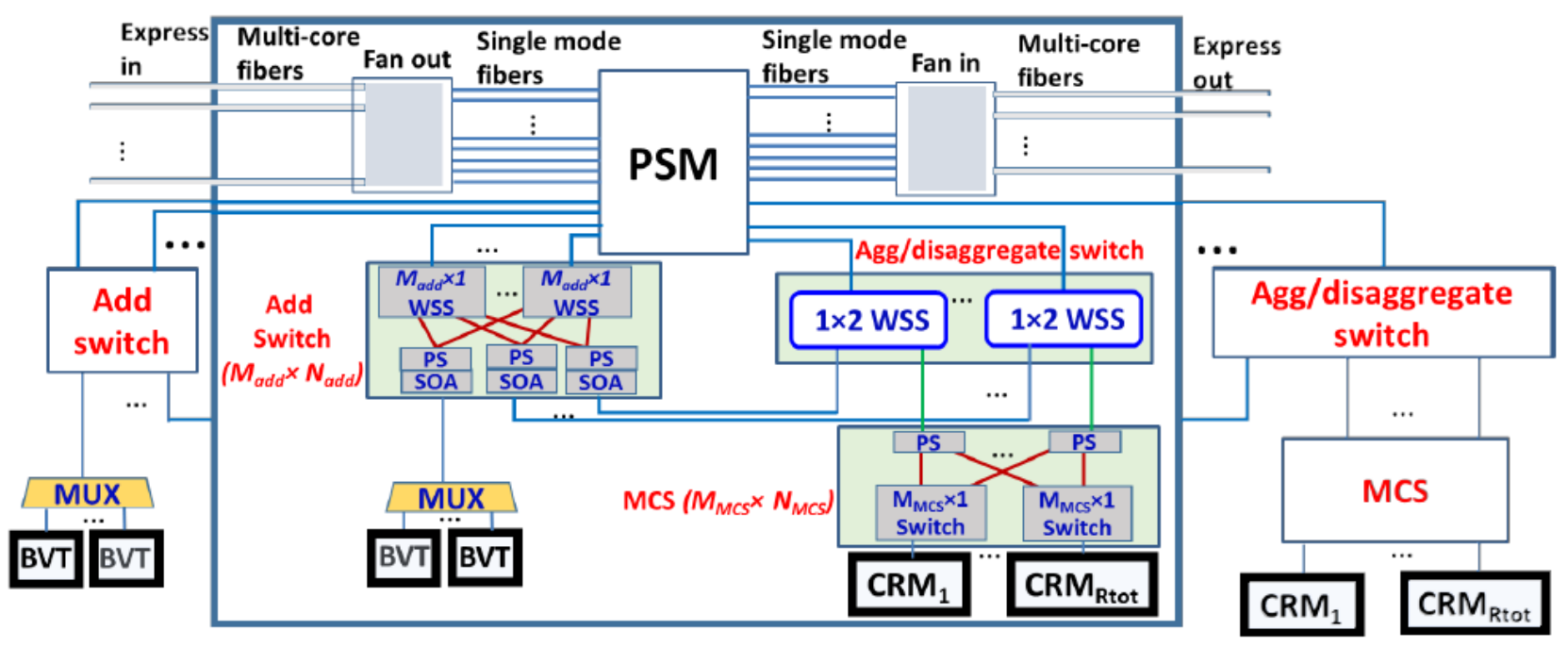

Figure 2: Schematic representation of a modular metro core node architecture with the photonic WDM switch functionalities.

\subsection{Metro Access Node architecture with edge computing and operation}

Recently optical metro node architecture based on optical "whitebox" concept, with distributed DC capabilities, i.e. using disaggregated hardware and local processing and storage resources, has been proposed [9-10]. The CORD architecture [10] includes a collection of commodity servers interconnected by a fabric constructed from electrical 
whitebox switches. The switching fabric is organized in a leaf and spine topology to optimize for east-west traffic between the access network that connects customers to the Central Office and then to the operator's core network. Here, we propose an optical metro node architecture exploiting the WDM cross - connects as shown in Fig. 3. It includes both optical and electrical (interfaces) switching to interface multiple access (PON, 5G, enterprise) to the optical network. Moreover, the optical node includes also a number of servers organized in racks (connected via an electronic ToR switch). As shown in Fig. 3, the interfaces and TORs are interconnected by a number of WDM cross-connect in a spine and leaf architecture. Each interface and TOR switch is equipped with a number of WDM bi-directional optical links. The main difference with CORD is the interconnect networks built by WDM cross-connect instead of electronic switches. This allows to switch transparently the data traffic between the interfaces and the TORs without expensive $\mathrm{O} / \mathrm{E} / \mathrm{O}$ converters, greatly reducing the power cons umption and costs. Moreover, the ability of the WDM cross -connect to switching in wavelength, space, and time (nanoseconds scale) allows for fully exploit statistical multiplexing. The number of interconnected interfaces and capacity depends on the radix of the switch and the amount of parallel WDM cross-connect. Part of the traffic belonging to the same access (same PON or same servers pool) is then directly exchange by the interfaces (or TORs). The other part of the traffic is directed to the network interface via multiple WDM transceivers, eventually with variable data rate and modulation formats. The multiple WDM transceivers allow to scale the bandwidth between the interfaces/ToRs and the WDM cross-connect network. The control plane is in charge of configuring the transceivers (data format and rate) and the WDM cross-connects.

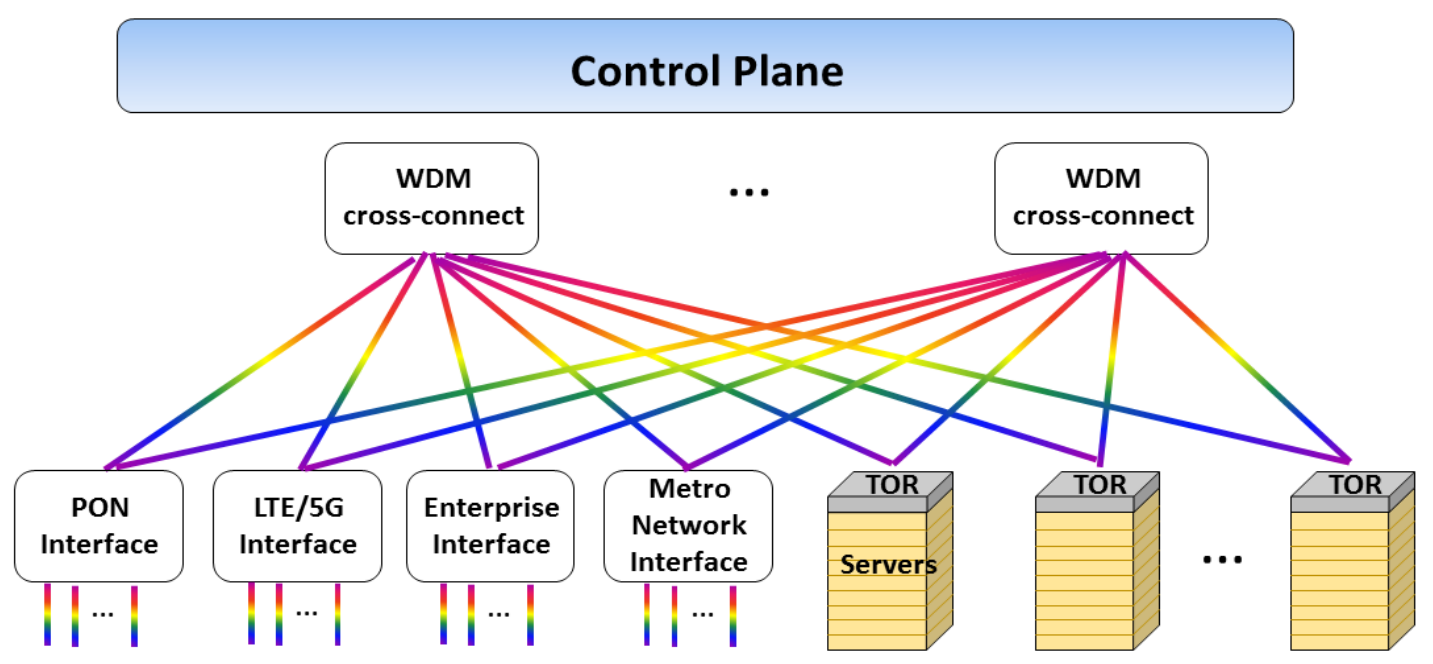

Figure 3: Schematic of the optical metro node with edge computing functionalities.

\subsection{Opical data center network architecture based on photonic WDM switches}

The DCN architecture based on WDM cross-connect switches is shown in Fig. 4. It consists of two parallel intra- and inter-cluster networks. An intra-cluster MxM optical switch interconnects the M ToRs. The inter-cluster network consists of $\mathrm{M}$ independent $\mathrm{NxN}$ optical switches that interconnect the ToRs of different clusters, namely the m-th optical switch interconnects the ToRm of each cluster, with $\mathrm{m}=1, . ., \mathrm{M}$. Fast flow control is implemented to solve packet contention due to the lack of optical buffer [11]. The advantages of this architecture are multiple. First, the parallel instead of hierarchical operation makes the DCN bounded in a flat way. Second, the scaling out of the ToRs goes as the square of the optical switch radix; by using $32 \times 32$ optical switches, such architecture could interconnect more than 1000 TORs (40000 servers). Third, the transparency to data rate and data format of the optical switch could allow for scaling up the data rate per link without changing the infrastructure, or adding more switching ports. Four, the sub-microseconds reconfiguration time of the optical switches allows to operate on long as well as on short flows exploiting statistical multiplexing. Five, no optical buffers are required, small electrical buffers at the TORs due to the short distance between the TORs allows an efficient implementation of the optical flow control to guarantee high performance of the DCN. Last, multiple paths are available which improve the fault tolerance and allow load balancing for further improving the performance of the DCN. Details on the operation and performance of this DCN architecture is reported in [11]. 


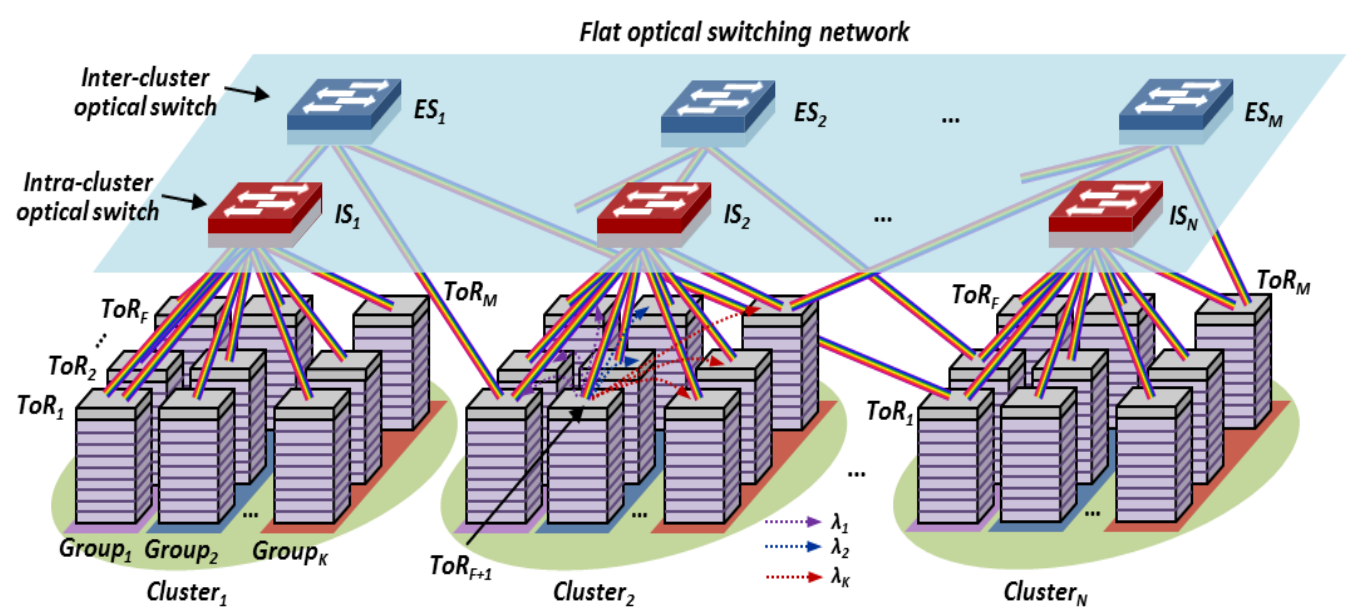

Figure 4: Intra-DCN network based on WDM cross connect switches.

\section{PHOTONIC INTEGRATED WDM SWITCHES}

The schematic of the optical wavelength, space, and time WDM cross-connect switch employed to realize the optical metro node architecture and the DCN architecture discussed above is illustrated in Fig. 5. The non-blocking optical cross-connect has $\mathrm{N}$ inputs, and each input can process $\mathrm{M}$ different wavelengths. The modular cross-connect processes the N WDM inputs in parallel by the respective optical modules, and forwards the individual wavelength channels to any output ports according to the wavelength channels to any output ports according to the switching control signals provided by the control plane. Each optical module consists of a 1:N splitter to broadcast the WDM channels to the $\mathrm{N}$ wavelength selective switches (WSS). The outputs of the N WSSs are connected to the $\mathrm{N}$ wavelength combiners of the respective N output ports. Each WSS can select one or more wavelength channels and forward the channels to the output

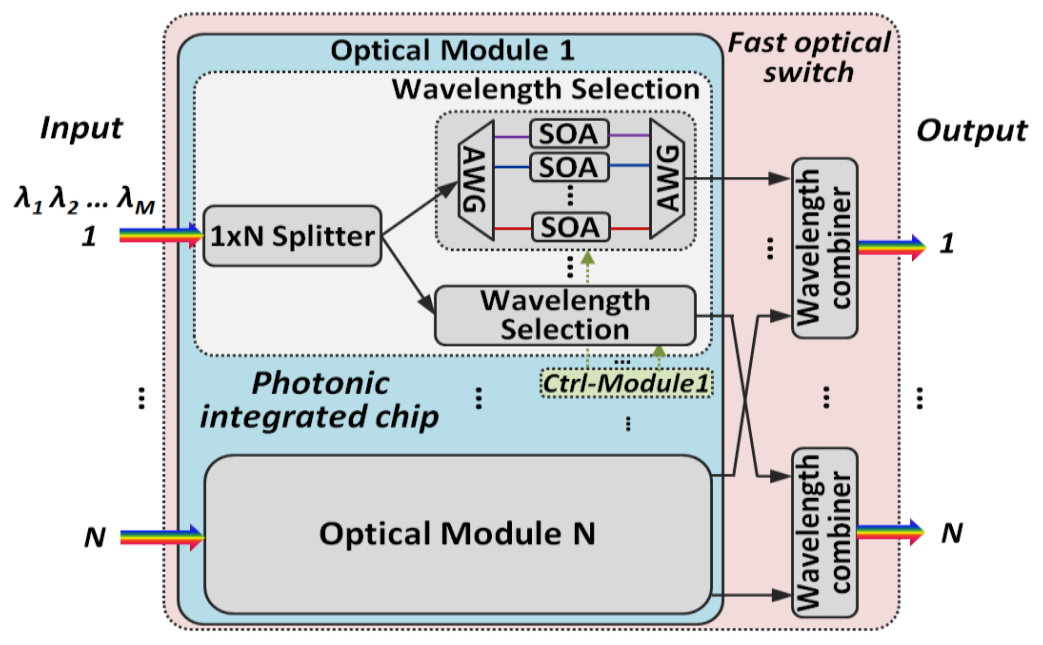

Figure 5: Schematic of the photonic WDM cross-connect switch.

ports according to the control signals. The WSS consists of two AWGs and M SOA based optical gates. Turning ON or OFF the M SOA optical gates determines which wavelength channel is forwarded to the output or is blocked. Multicast operation is also possible with this architecture. The broadband operation of the SOA enables the selection of any wavelength in the $\mathrm{C}$ band. Moreover, the amplification provided by the SOA compensates the losses introduced by the two AWGs. Scaling the port-count leads to a linear increase in components and energy consumption, by employing copies of the identical modules. Based on the schematic shown in Fig. 5, a PIC integrating four optical modules each 
with four WDM channels (without wavelength combiner) is fabricated and shown in Fig. 6. The chip was realized in a multi-project wafer (Jeppix platform). More details of the chip are reported in [12]. Switching operation for highcapacity and multi-level modulated traffic in DCNs (50 Gb/s NRZ-OOK, $20 \mathrm{~Gb} / \mathrm{s}$ PAM4 and >30 Gb/s DMT) has been reported in [13].

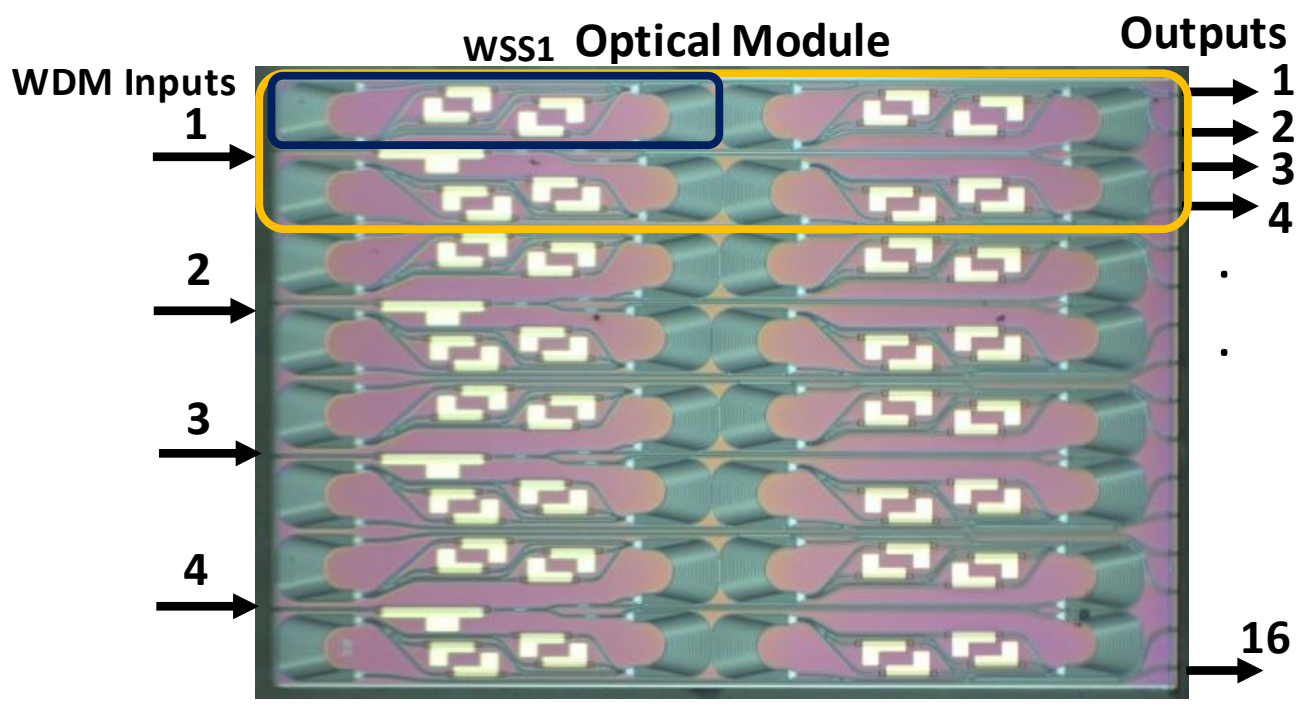

Figure 6: Photonic integrated WDM cross-connect switch.

The modular photonic integrated WDM switch reported in Fig. 6 can switch 4 WDM channel for each of the 4 input port. To further study the scalability of the proposed switch architecture, we have designed and fabricated a photonic integrated 1x8 WDM switch module, so that the new device can process $8 \mathrm{WDM}$ channel for each of the 8 input ports. We report here the experimental assessment of the switching performance of a photonic integrated $1 \times 8 \mathrm{WDM}$ switch module with $8 \mathrm{WDM}$ channels at 10,20, $40 \mathrm{~Gb} / \mathrm{s}$ NRZ-OOK data.

Figure 7 shows the photonic chip with two optical modules. Each module consists of a 1:2 MMI splitter to broadcast the WDM channels to both the WSS that process one of the WDM inputs in parallel. The WSS consists of an SOA booster $(1 \mathrm{~mm})$ at the input, two AWGs, 8 quantum well active InGaAsP/InP SOA based optical gates $(950 \mu \mathrm{m})$, and the SOA as pre-amplifier at the output $(2 \mathrm{~mm})$. The first $1 \times 8 \mathrm{AWG}$ operates as wavelength de-multiplexer. Turning on or off the 8 SOA optical gates determines which wavelength channel is forwarded to the output or is blocked. The second 8x1 AWG operates as wavelength multiplexer. The AWGs are designed with a free spectral range (FSR) of $2 \mathrm{~nm}$. Multicast operation is also possible with this architecture. The broadband operation of the SOA enables the selection of any wavelength in the $\mathrm{C}$ band. Moreover, the amplification provided by the SOA s compensates the losses introduced by the two AWGs. The chip has been realized in a multi-project wafer (MPW) with limited space of the cell (4.6 $\mathrm{mm} \times 4 \mathrm{~mm}$ ).

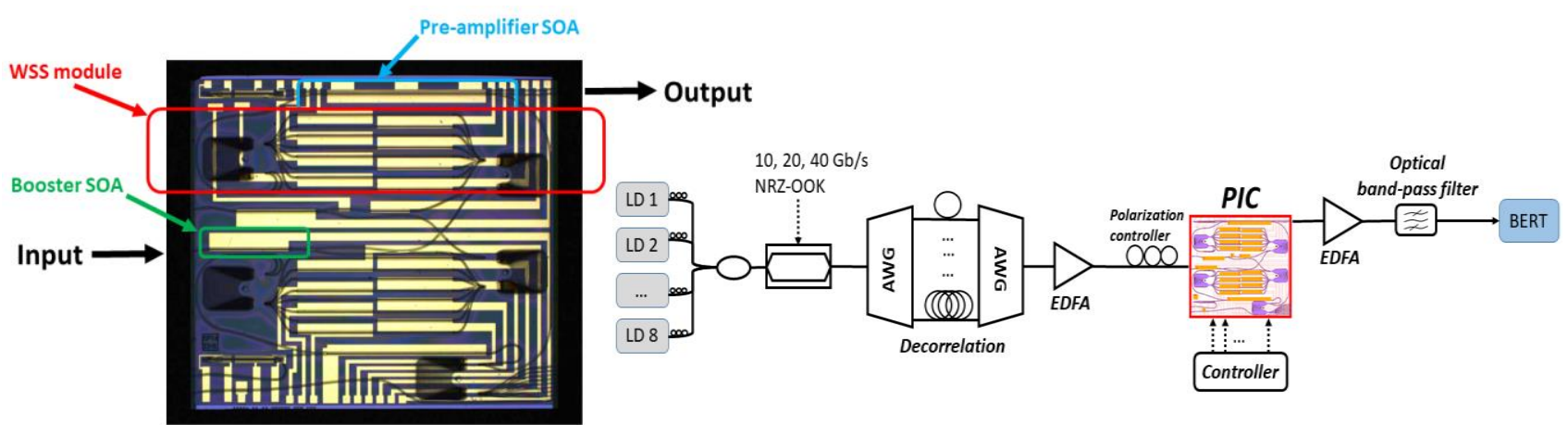

Figure 7: Microscope image of the modular WDM photonic switch (left). Experimental set-up employed to assess the performance of the WDM photonic cross-connect. PIC (Photonic Integrated Chip), LD: Laser Driver, BERT: BER tester (right). 


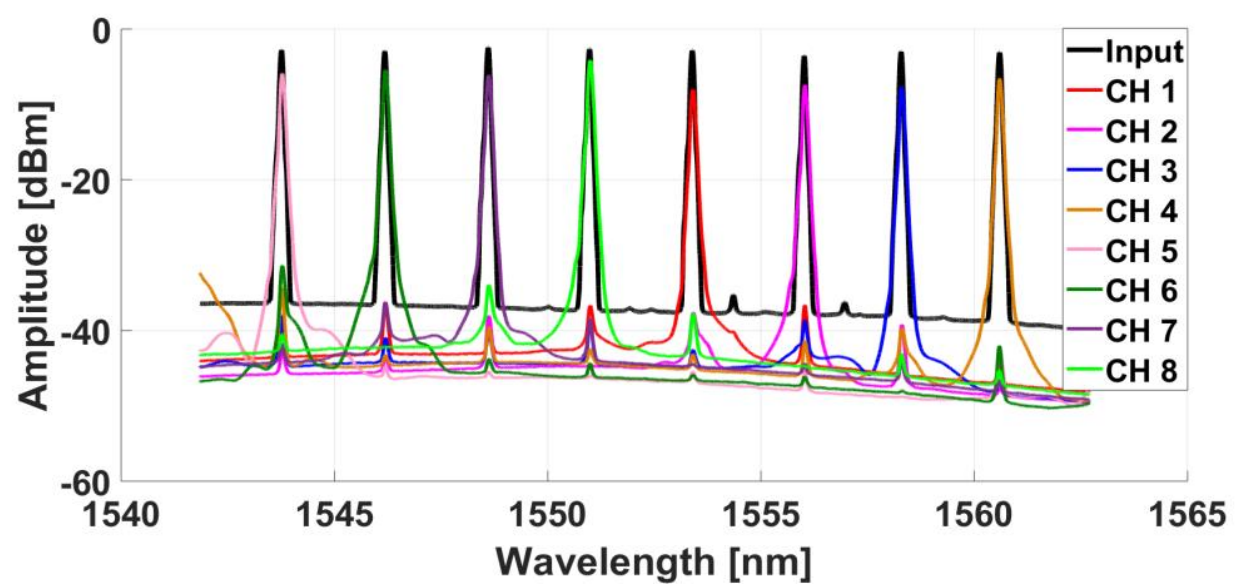

Figure 8: Optical WDM input and eight switched output channels.

An image of the photonic integrated switch is shown in Fig. 7 (left) with colored boxes around the main components and one of the two WSS modules contained in the photonic chip. The light shaded electrodes are routed through on-chip metal tracks and then wire bonded to the neighboring PCBs to enable the control of the SOA gates. Lensed fibers have been employed to couple the light in and out of the chip. The experimental set-up employed to assess the 1x8 WDM WSS photonic switch module is shown in Fig. 7 (right). Eight optical input channels spaced by $2 \mathrm{~nm}$, from $\lambda 1=1544 \mathrm{~nm}$ to $\lambda 4=1560 \mathrm{~nm}$, are generated and coupled together. The WDM signal was modulated using an amplitude modulator driven by $10,20,40 \mathrm{~Gb} / \mathrm{s}$ pattern generator with $2^{31}-1$ PRBS for $10,20 \mathrm{~Gb} / \mathrm{s}$, and $2^{7}-1$ PRBS for $40 \mathrm{~Gb} / \mathrm{s}$. Since a single modulator has been used for all the 8 channels, a de-correlator consisting of two AWGs and delay lines has been used to de-correlate the channels. The eight WDM channels are de-correlated and launched into the input of the photonic chip. As the optical modules are identical, we have assessed the operation of one optical module to characterize the switching operation, the upper one. The optical power of the WDM input channels was $-3 \mathrm{dBm} /$ channel. Figure 8 shows the input WDM and the outputs spectra. Considering the coupling loss of around $12 \mathrm{~dB}$, on cheap gain is clearly visible, and thus lossless operation. The input and output booster SOAs were biased with currents 80-98 mA and 90-97 mA, respectively. The temperature of the chip was maintained at $20^{\circ} \mathrm{C}$ through a water cooler. Polarization controllers were employed at the input of the chip as well as per each channel (LD 1 to LD 8). First, we assess the static operation of the WSS. One WDM channel at the time is statically switched at the WSS output by enabling one of the eight SOA gates at the time. The current applied at each SOA gate was $80-98 \mathrm{~mA}$. To investigate the data integrity of the chip operation, BER curves are recorded and shown along with eye diagrams in Fig. 9 for 10, $20 \mathrm{~Gb} / \mathrm{s}$.

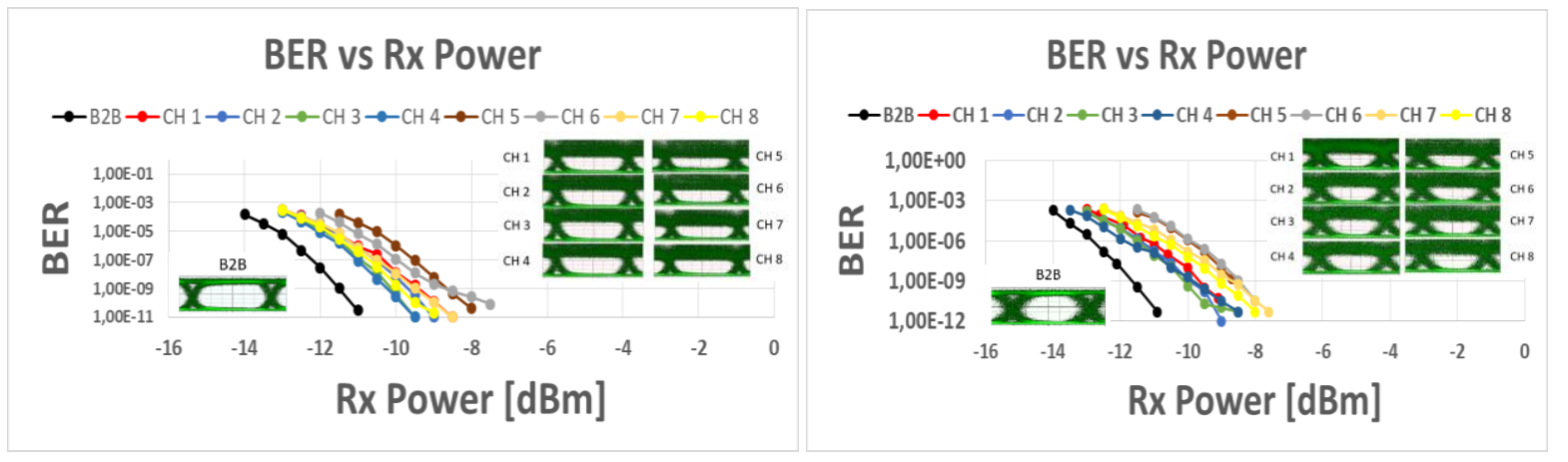

Figure 9: BER vs Received Optical Power curves and eye diagrams of switched channels at the output port for WDM input signal for $10 \mathrm{~Gb} / \mathrm{s}$ (left) and $20 \mathrm{~Gb} / \mathrm{s}$ (right), NRZ-OOK traffic.

Figure 9 shows clear open eye diagrams of the switched channels. The BER curves, including the back-to-back as reference, are also shown in Figure 9. Error-free operation with a penalty less than $3 \mathrm{~dB}$ was measured at BER=1e-09. The main source of this penalty is the limited OSNR input due to multiple amplifications to compensate multiplexers, optical modulators and decorrelator at the transmitter side, the fiber to chip coupling losses, and thus the accumulated 
noise on the signals through the chip due to the SOAs. Packaging of the chip will reduce the los ses and thus improve the OSNR and reduce the penalty. Additionally, in order to stress further the performance of the switch in terms of data rate, measurements of penalty have also been conducted for $40 \mathrm{~Gb} / \mathrm{s}$. Due to the limited OSNR of the channels at the input and output, it was not possible to achieve the error-free quality for the received signal. Nonetheless, some measurements with $40 \mathrm{~Gb} / \mathrm{s}$ traffic were attempted by modulating only one of the 8 channels at a time and recoupling it with the other 7 optical carriers before entering the chip in a WDM form. The BER curves for this situation are shown in Fig. 10 (left). Error-free operation with a penalty less than $2 \mathrm{~dB}$ was measured at $B E R=1 \mathrm{e}-09$. The results of this measurements show that the switching capabilities of the chip were good enough for switching the WDM channels modulated at $40 \mathrm{~Gb} / \mathrm{s}$ demonstrating that the OSNR of the signal is the main impediment for higher bit rates.

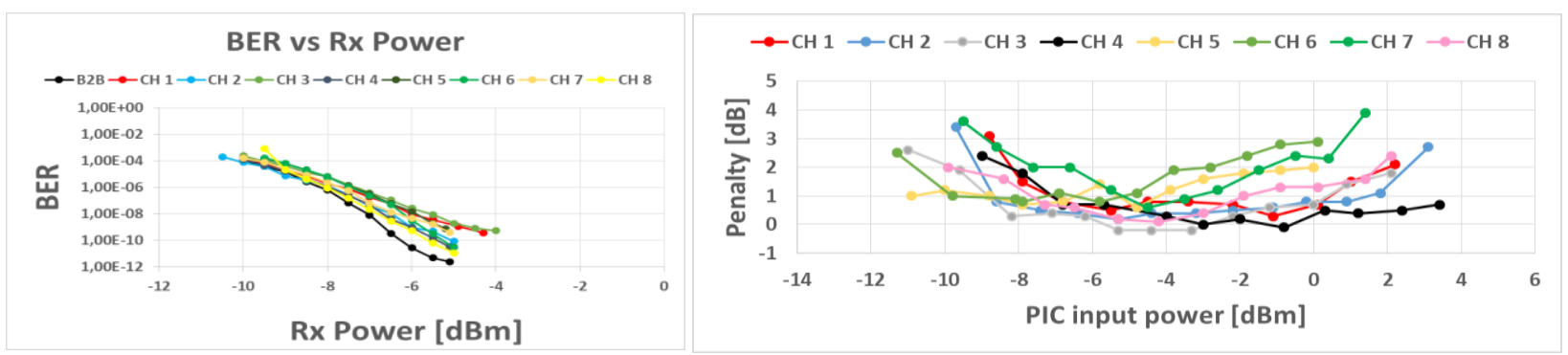

Figure 10: BER curves of modulated channels switched at the output port for WDM input signal for $40 \mathrm{~Gb} / \mathrm{s}$ NRZ-OOK traffic (left). Power dynamic range for single channel $40 \mathrm{~Gb} / \mathrm{s}$ NRZ-OOK traffic (right).

To show the potential of the optical switch to scale to a larger number of ports the measurements of the power dynamic range were conducted for single channel NRZ-OOK $40 \mathrm{~Gb} / \mathrm{s}$. For these measurements the power penalty guaranteeing a BER $=1 \mathrm{e}-09$ of the signal at the chip output was recorded for different input optical powers of the channels. The currents of the booster and gate SOAs on chip were varied to keep an equalized output power. The results are shown in Fig. 10 (right). As seen from the graphic the optical switch presents a dynamic range $>8 \mathrm{~dB}$ within a $3 \mathrm{~dB}$ power penalty for all the channels. Lower input power is limited by the OSNR degradation where the noise is more dominant. Larger penalty is also found for higher input power due to the increased sensitivity to saturation. The large dynamic range operation indicates that a WDM WSS with larger port count is possible.

\section{EXPERIMENTAL ASSESSMENT OF METRO CORE NODE}

The experimental setup validating the operation of the metro core node shown in Fig. 2 and exploiting the photonic WDM switch is shown in Fig. 11a. The input traffic to the metro core node is generated by six WDM transmitters at 40 $\mathrm{Gb} / \mathrm{s}$ (Tx). The 4 output ports of a fully reconfigurable $1 \mathrm{x} 4 \mathrm{WSS}$ was employed to emulate a MCF and to dynamically assign the WDM channels to two different input ports of the PSM, namely $\lambda 1$ and $\lambda 2$ to I-1, and $\lambda 3$ and $\lambda 4$ to I- 2 . The channels $\lambda 5$, and $\lambda 6$ were used as add channel emulating traffic generated by the local BVT. The port I-3 of the PSM is used for the add traffic (wavelengths shown in red). The output port O-1 and O-2 of the PSM are used as express port, while O-4 as drop port. The PSM is based on MEMS technology, but it can be replaced by photonic integrated chips. The aggregate/disaggregate switch is based on a wavelength blocker with (de)multiplexing AWGs and SOA gates as shown in the functional diagram in Fig. 11(b). Due to the presence of multiple optical module with WSS integrated on the same photonic chip (an inherent $1 \times 4$ splitter) is used to emulate the MCS functionality. The microscopic picture of 4 modules of the WSS with 4 inputs and 16 outputs is shown in Fig. 11(c); the employed WSS for the aggregate/disaggregate switch in this experiment is WSS-1. The details of the has been discussed in section 3 . Of the two input wavelengths, $\lambda 1$ and $\lambda 2, \lambda 1$ is locally dropped while $\lambda 2$ is aggregated with $\lambda 5$ and $\lambda 6$ by an Add switch. The Add switch is replaced by a power combiner (PC) in this experiment due to the availability of a single WSS chip. The added wavelengths are sent out as express traffic at $\mathrm{O} 2$ of the PSM. The optical spectrum at drop port and aggregate port of the WSS are given in Fig. 11(d) and 11(e), respectively. Afterwards, BER measurements are conducted for 40 Gbps and 20 Gbps OOK NRZ data at different point in the set-up: a: back-to-back, b: express traffic after the switch, c: local drop traffic and d: aggregated traffic. Fig. 12(a) shows the BER measurement for $\lambda 1$ and $\lambda 2$ at $1536.8 \mathrm{~nm}$ and $1540 \mathrm{~nm}$ for measurement points $\mathrm{c}$ and $\mathrm{d}$ indicated in Fig. 11(a), respectively. A power penalty of only $1 \mathrm{~dB}$ is incurred in case of the aggregated wavelength $\lambda 2=1540 \mathrm{~nm}$ at $20 \mathrm{~Gb} / \mathrm{s}$ and $1.5 \mathrm{~dB}$ for $40 \mathrm{~Gb} / \mathrm{s}$ data. The power penalty increases to $3.5 \mathrm{dBs}$ in 


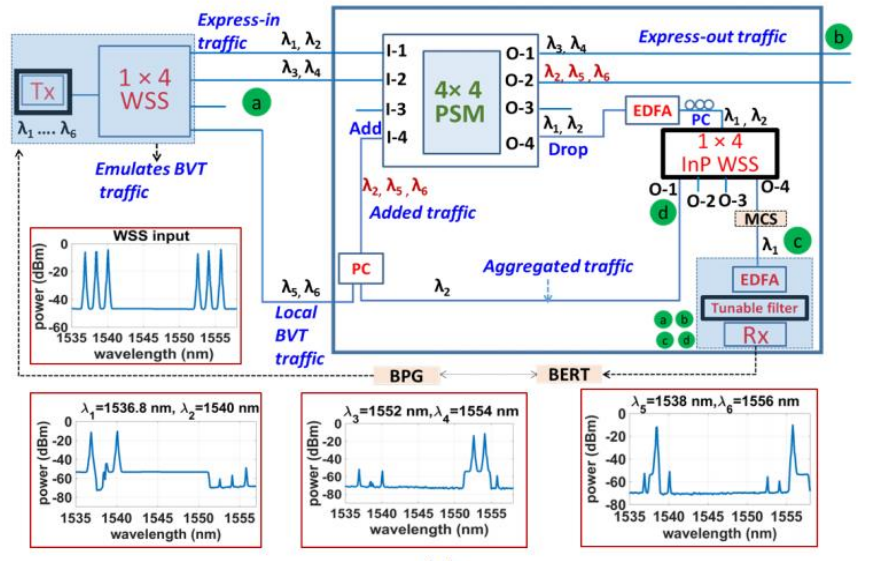

(a)

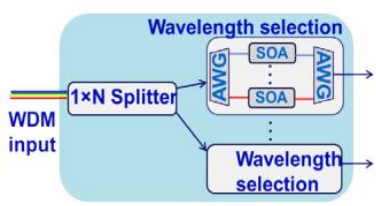

(b)

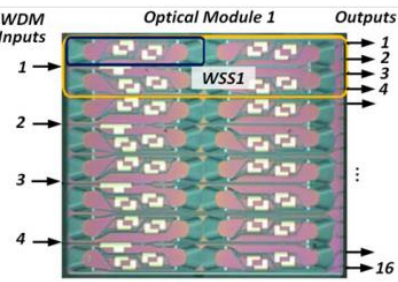

(c)

Figure 11: (a) Experimental Setup, (b) WSS functional representation (c) microscopic picture. Dropped traffic at different point in the setup.

case of dropped $\lambda 1=1536.8 \mathrm{~nm}$ because of the extra path loss in O-4 of WSS (unpackaged devices with large fiber-tochip coupler have been employed in the experiments). Fig. 12(b) shows the BER measurement at express out traffic of MEMS-O1, point $b,(\lambda 3: 1552 \mathrm{~nm}, \lambda 4: 1554 \mathrm{~nm})$ incurring penalties of less than $1 \mathrm{~dB}$ at both data rates. BER measurements for added wavelengths at express port MEMS-O2 are given in Fig. 12(c). Power penalties for BVT wavelengths $\lambda 5: 1538 \mathrm{~nm}$ and $\lambda 6: 1556 \mathrm{~nm}$ is less than $1 \mathrm{~dB}$. The power penalty increases to $1.8 \mathrm{~dB}$ for $\lambda 2=1540 \mathrm{~nm}$ since it passes through the PMS twice and WSS once. Those results experimentally verify the novel metro core node functionality in the context of a modular architecture as applicable in a high capacity metro core network.

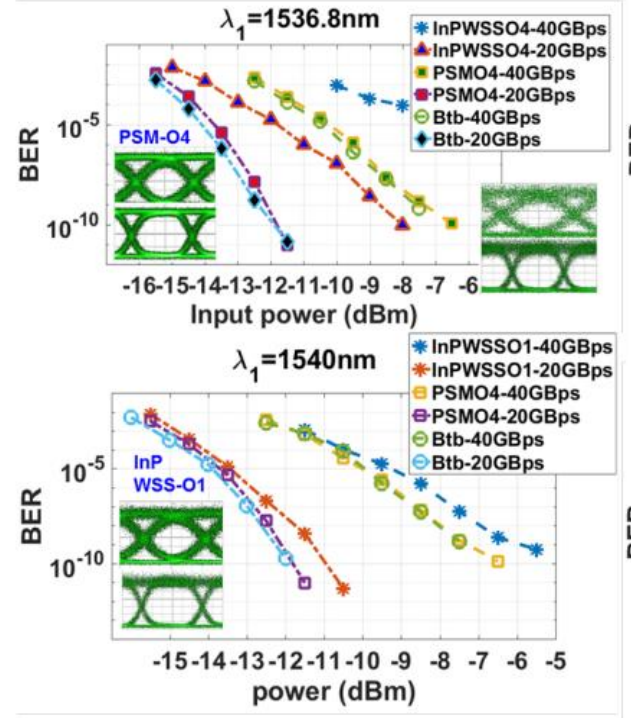

(a)

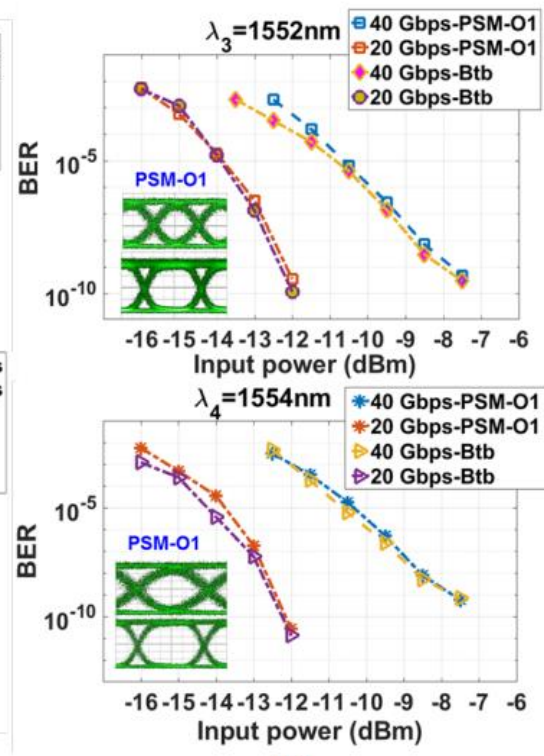

(b)

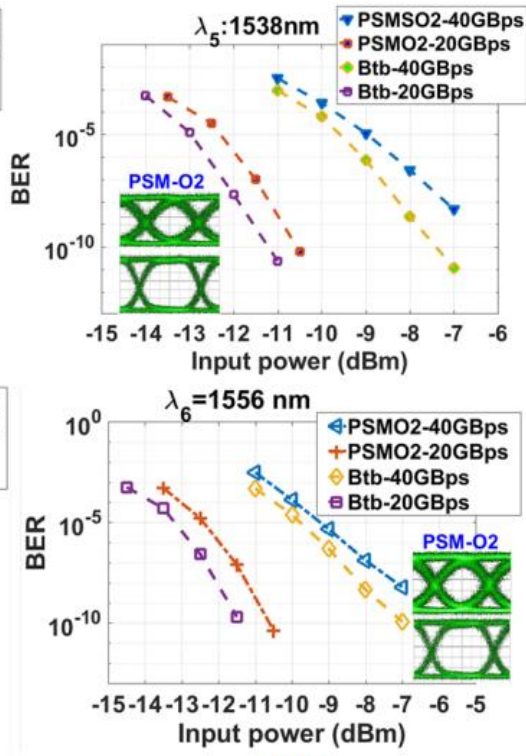

(c)

Figure 12: (a) BER measurement for the drop traffic WSSO-1 (b) BER measurements at Express out traffic at PSM-O1 (c) BER measurements at Express out traffic at PSM-O2.

\section{CONCLUSIONS}

We have presented a novel modular optical metro core and metro access node architectures employing a photonic WDM cross-connect switches. The metro core node consists of express and add/drop ports, photonic integrated WSS aggregation/disaggregation functions for merging/dropping the network traffic, and photonic integrated multi-cast switch (MCS). The modular approach enables to scale the architecture in a pay-as-you-grow approach by using photonic integrated devices for wavelength and space switching. Additionally, a low-cost 2-degree photonic integrated mini- 
ROADMs for dynamic re-configurable metro-access nodes in a ring topology will be presented. The photonic WDM switches are based on InP technology and employ semiconductor optical amplifiers (SOA) as on -chip gain element for lossless operation. Moreover, the fast switching time of the SOA and the high contrast ratio make the SOA a good candidate for fast packet-based switching operation. The capability of the photonic WDM switches to transparently process data signals in wavelength, space, and time allows for high dynamic, flexible, and scalable interconnected networks.

\section{ACKNOWLEDGEMENTS}

The authors would like to thank the EU H2020 PASSION project ( $\left.n^{\circ} 780326\right)$, the EU H2020 QAMELEON project ( ${ }^{\circ}$ 780354) for supporting this work.

\section{REFERENCES}

[1] M. Maternia, et al. 5G PPP use cases and performance evaluation models. [On-line] Available: https://5gppp.eu

[2] B. Ušćumlić, et al. Optimal dimensioning of the WDM unidirectional ECOFRAME optical packet ring, Photonic network communications, 2011, 22(3): 254-265.

[3] C. Simonneau, et al. Packet-optical Add/Drop multiplexer technology: A pragmatic way to introduce optical packet switching in the next generation of metro networks, Proc. LEOS, 2009.

[4] W. Miao, et al. Low Latency Optical Label Switched Add-Drop Node for Multi-Tb/s Data Center Interconnect Metro Networks. Proc. ECOC 2016.

[5] M. Ruffini,et. al., "Access and Metro Network Convergence for Flexible End-to-End Network Design [Invited]," JOCN, 524-535 (2017)

[6] J. Fernandez-Palacios "Telefonica Optical SDN strategy \& use cases" Workshop "Photonic transport systems for $5 \mathrm{G}$ networks, ECOC (2018)

[7] S. Sarmiento, et.al, "Cost-effective DWDM ROADM design for flexible sustainable optical metro-access networks," JOCN (2017).

[8] M. Garrich et al., "Experimental demonstration of function programmable add/drop architecture for ROADMs" JOCN, 7(2), (2015).

[9] M. De Leenheer et al., http://sdn.ieee.org/news letter/ january-2016/rethinking-metro-networks.

[10] M. De Leenheer et al, OFC 2016, OSA Tech. Digest.

[11] W. Miao et al., JLT, vol. 34, no. 17, pp. 4066-4075 (2016)

[12] N. Calabretta et al, ECOC 2016, Paper W.2.F.3.

[13] N. Calabretta et al, Optical Fiber Communications Conference and Exhibition, OFC 2017, Tu3F.1, 2017. 